\title{
The Major Metaphors of Evolution: Visualizing the Extended Synthesis
}

\author{
Daniel R. Brooks
}

Published online: 6 September 2011

(C) Springer Science+Business Media, LLC 2011

Scientists have odious manners, except when you prop up their theories. Then you can borrow money off them. -Mark Twain

Scientists, when dealing with their normative frameworks (paradigms, pet theories), use two kinds of language: nomenclature (labels) and metaphor. The technical language of nomenclature attempts to eliminate ambiguity in concepts and entities within an area of science. An excellent example in Biology is the use of scientific names for species. If a North American and a European call a bird a "robin," they are referring to two different species that are not even closely related. If, however, they both say Turdus migratorius, there is no confusion. Choosing a "dead language" for formal nomenclature helps preclude additional meanings creeping into the nomenclatural designations. Scientists who are comfortable with a given normative framework tend to embrace nomenclature as the solution to problems, which they see as mostly a matter of reducing ambiguity within a theoretical framework assumed to be fundamentally true. Those scientists tend to mistrust metaphors because they allow too many possibilities, thus introducing ambiguity into the framework.

Scientific change is creative. When there is need, or desire, to make a change in a normative framework, therefore, metaphor becomes the language of choice. Perhaps the most important function of metaphor in science is in extending existing nomenclature to accommodate new concepts and

D. R. Brooks $(\bowtie)$

Department of Ecology \& Evolutionary Biology,

University of Toronto,

Toronto, ON, Canada M5S 3G5

e-mail: dan.brooks@utoronto.ca empirical findings. This is contrary to the normative use of nomenclature, and yet it is essential. If we created new nomenclature for each new proposal, there would be no way to show connections between the old and the new frameworks. Metaphors give people a reason to learn new ideas and are the means by which people learn those new ideas. Metaphors also present natural truths using language that allows understanding by non-specialists. Darwin's literary skills were key to the great success of Origin of Species among non-specialists. Darwin was a master at linking common everyday experiences and knowledge with technical observations by biologists, linking them together metaphorically to produce his panoramic synthesis of the history of life.

As Darwinism became widely accepted, the language of evolution changed from metaphor to nomenclature, with a narrowing of perspective. Neo-Darwinism, or the synthetic theory of evolution, is currently the primary normative framework for evolutionary biology. I have suggested that neo-Darwinism is not a refined and upgraded version of Darwinism, a narrowing of vision with almost exclusive focus on only one of Darwin's metaphors, natural selection. Darwinism differs in a number of important respects from neo-Darwinism, enough to warrant calling them different normative frameworks. Furthermore, I have argued that an Expanded Synthesis should not be seen as an expansion of neo-Darwinism, which has "expanded" as much as it can, but should be viewed as a return to Darwinism and an expansion of that framework (Brooks 2011a, b, c).

My goal in being provocative has been to make readers feel as ambivalent about their own views of evolution as I have felt about mine. I am not calling for a kind of ecclesiastical dialogue in which each side expects the other to convert at 
some point. Rather, I am asking us to learn about how we think about evolution by learning how others see our own normative views (Fisch and Benbaji 2011). Metaphor is essential for this. Fortunately for my "back to the future" theme, metaphors cannot disappear-forgotten or set aside, they can always be recovered and reexamined in light of new information or challenges to an existing framework. So, in this contribution, I return to the metaphors elaborated by Darwin and neglected during the development and "hardening" of the New Synthesis. Then I show how those metaphors provide a common link among some late twentieth century proposals. Finally, I suggest some new generalized metaphors aimed toward allowing researchers to connect Darwin's full view of life and the progressive proposals from the 1980s and 1990s with the Extended Synthesis - in whatever form ultimately emerges.

\section{Darwin's Forgotten Metaphors}

Natural selection, Darwin's most novel metaphor, was the key concept in distinguishing Darwinian selectionism from Lamarckian adaptationism. Darwin therefore devoted the bulk of Origin of Species to elaborating the concept. As well, even though Darwin viewed natural selection as an emergent property of the nature of the organism, mechanisms underlying the inherent properties of organisms were not understood (Darwin referred to the mysterious laws of growth, the laws of inheritance, and the laws of the correlation of parts without specifying mechanisms to explain them). As a result, natural selection was the concept most amenable to normalizing language and became the primary focus of neo-Darwinism.

\section{The Conditions of Existence}

Darwin proposed that biological diversity emerged from

...two factors: namely, the nature of the organism and the nature of the conditions. The former seems to be much more the important; for nearly similar variations sometimes arise under, as far as we can judge, dissimilar conditions; and, on the other hand, dissimilar variations arise under conditions which appear to be nearly uniform. (Darwin 1872: 32)

Darwin's conception of the "nature of the organism" was explicit, even if he lacked mechanisms of inheritance and ontogeny. Darwin understood that organisms were historically and developmentally cohesive wholes, and therefore it was in the nature of the organism to produce offspring that were all highly similar (but not identical) to each other and to their parents and other ancestors. He also postulated that reproduction produced variation without regard for envi- ronmental conditions, and therefore it was in the nature of the organism to produce these offspring in numbers far exceeding the resources available for their support. This was Darwin's Necessary Misfit (Brooks and Hoberg 2007; Brooks 2011a, b).

Organisms cannot produce more offspring than there are resources to support them in a Panglossian (Lamarckian) world, so there must be constraints on responses to the surroundings. Darwin resolved this conundrum by postulating that the nature of the organism, in the form of insensitivity to the nature of the conditions, created the constraints. And yet, those constraints were not absolute. All surviving organisms have positive Darwinian fitness, but some are fitter than others in the environment in which they were produced. These tend to predominate numerically over their merely adequate relatives. Those relatives, however, survive and play a decisive role in Darwinian evolution. Whenever an environment changes, the fittest organisms in the old environment might not survive at all in the new, whereas some of the merely adequate in the old environment might have the adaptations necessary to survive, and even flourish, in the new one. Natural selection was thus an emergent property of the inevitable conflict created by the conditions of existence and was a metaphor for the ways to resolve such conflicts, setting the stage for resolution of conflicts yet to come. More than 150 pages after introducing the duality of the conditions of existence, Darwin called it the higher law of biology, underscoring the emergent nature of natural selection:

It is generally acknowledged that all organic beings have been formed on two great laws - unity of type and the conditions of existence.... On my theory, unity of type is explained by unity of descent. The expression [my italics] of conditions of existence...is fully embraced by the principle of natural selection.... Hence in fact the law of the Conditions of Existence [my italics] is the higher law; as it includes, through the inheritance of former adaptations, that of Unity of Type. (Darwin 1872: 194-195)

\section{The Complexity of Evolution}

Darwin proposed two rich visual metaphors to help readers understand the fundamental complexity of evolution.

\section{The Phylogenetic Tree}

As buds give rise by growth to fresh buds, and these, if vigorous, branch out and overtop on all sides many a feebler branch, so by generation I believe it has been with the great Tree of Life, which fills with its dead and broken branches the crust of the earth, and 
covers the surface with its ever-branching and beautiful ramifications. (Darwin 1872: 000-000)

The Tree of Life metaphor is more than an accounting scheme; it is a symbol of a major part of the evolutionary process. Living systems are capable of acting in their own behalf, but more importantly, they regularly take the initiative, using what they have inherited. Metaphorically, the present is the state in which biological systems create their own futures based on their own pasts. Organisms carry so much of their history with them that most explanations for their appearance and function stem from their past - this is the focus of historical ecology (Brooks and McLennan 2002). Specific points of origin in space and time play integral roles in explaining the properties of species and the organisms that comprise them, most importantly how they interact with their surroundings, including other species. I recently spent a year in Europe, where a "sycamore" is a maple (Acer pseudoplatanus) and a "plane tree" (Platanus orientalis) is what I call "sycamore" (Platanus occidentalis). Darwin's metaphor of natural classification being a phylogeny enables us to understand why North American sycamores and European plane trees resemble each other so closely, why their ecological preferences are so similar, and why they are able to hybridize so readily.

Darwin's phylogenetic tree metaphor contrasted with a progressive view of diversity embodied in the Scala naturae, in which "lower forms" were replaced by "higher forms." Thus, the only illustrated metaphor Darwin ever provided in any edition of Origin of Species specifically underscored the notion of evolution as one of selective accumulation of diversity rather than selective replacement.

\section{The Tangled Bank}

It is interesting to contemplate a tangled bank, clothed with many plants of many kinds, with birds singing on the bushes, with various insects flitting about, and with worms crawling through the damp earth, and to reflect that these elaborately constructed forms, so different from each other, and dependent upon each other in so complex a manner, have all been produced by laws acting around us. These laws, taken in the largest sense, being Growth with reproduction; Inheritance which is almost implied by reproduction; Variability from the indirect and direct action of the conditions of life, and from use and disuse; a Ratio of Increase so high as to lead to a Struggle for Life, and as a consequence to Natural Selection, entailing Divergence of Character and the Extinction of less improved forms. Thus, from the war of nature, from famine and death, the most exalted object which we are capable of conceiving, namely, the production of the higher animals, directly follows. There is grandeur in this view of life, with its several powers, having been originally breathed by the Creator into a few forms or into one; and that, whilst this planet has gone circling on according to the fixed law of gravity, from so simple a beginning endless forms most beautiful and most wonderful have been, and are being evolved.

(Darwin 1872: 000-000)

This is clear and a lyrically metaphorical statement which evokes visions of selective accumulation of diversity producing complex ecosystems. It also explicitly underscores Darwin's view that natural selection is an emergent property, reinforcing his conception of the Law of the Conditions of Existence.

\section{Reclaiming the Metaphors}

My personal rediscovery of Darwin's metaphors allowed me initially to understand the distinction between Darwinism and neo-Darwinism and the manner in which I had conflated these two very different theories, confusing myself and colleagues. Those metaphors then allowed me to understand connections between my proposals and those of other colleagues in the 1980s and 1990s. Those proposals were met with skepticism (and often hostility) by prominent neo-Darwinians who also believed that neoDarwinism was simply upgraded and modernized Darwinism. Those positions are logically incompatible, given the following sample summaries: Eldredge and colleagues (The Hierarchy Metaphor): Biological diversity is embodied in The Genealogical Hierarchy, a hierarchy of replicators and information flow (nature of the organism; tree of life); and the Ecological Hierarchy, a hierarchy of interactors and energy flow (nature of the conditions; tangled bank). Brooks and Wiley and colleagues (The Entropy and Complexity Metaphor): Evolution results from entropic increase in biological information, constrained by intrinsic (nature of the organism; tree of life) and extrinsic (nature of the conditions; tangled bank) cohesive properties. Maynard Smith and Szathmary and colleagues (The Major Transitions Metaphor): Major Transitions in evolution increase the efficiency of storing and transmitting information (nature of the organism; tree of life), thereby enhancing organism/environment interactions (nature of the conditions; tangled bank) leading to conflict resolution. In all three cases, evolution leads to selective accumulation of diversity.

The psychological need to maintain existing normative frameworks seems to be strong. Two recent volumes (McShea and Brandon 2011; Calcott and Sterelny 2011) attempt to normalize much of the above proposals within neo-Darwinism. I believe they failed because neo-Darwinism lacks the appropriate metaphors to accommodate these "back to the 
future" proposals. These volumes did, however, successfully underscore the strong differences between Darwinism and neo-Darwinism as normative frameworks.

I believe that the "Major Transitions" perspective is the most appropriate framework for moving forward to the Extended Synthesis. The initial books (Maynard Smith and Szathmàry 1995, 1999) were written in a normative style. However consistent with, and based on, Darwin's metaphors these proposals have been, they must be generalized even more to unify biology so we can go from the origin of life to today's diversity and back smoothly, and can move from biology to chemistry and physics and back smoothly. Molecular biology and systems chemistry, computers/informatics and exobiology all demand that we update and expand our metaphorical base. Therefore, I next suggest three categories of metaphors designed to link Darwin's views, the views of the late twentieth century thinkers encompassed by the "hierarchy," "complexity and entropy," and "major transitions" groups, along with more recent proposals and research programs. I hope this will be the beginning of a lively, interesting, and fun-filled discussion about evolution and metaphor.

\section{The Major Metaphors of Evolution}

\section{Time}

We're all children of Time. -Shevek, The Dispossessed (LeGuin 1974: 385)

No aspect of the Brooks and Wiley proposal created a more negative emotion than the assumption of inherent irreversibility in evolution. This included some decidedly non-progressive phenomena, such as aging and death, extinction, and the persistence of traits and organisms in environments where they did not arise and are not optimally adapted. For neo-Darwinism, irreversible phenomena must result from progressive ascendancy (ratchet irreversibility), environmental selection driving the system toward a better future. If at some point the next step to a better future requires retracing steps, selection ratchets that as well. Only time is inherently irreversible, and we treat that as something we get for free. But if thermodynamics and the legacy of John Maynard Smith have told us anything, it's that there's no free lunch in the universeeven time must be paid for.

Biological systems "buy" or "make" time by what I call the Shevek metaphor, for the main character in Ursula LeGuin's novel The Dispossessed. As I've detailed previously (Brooks 2011c), irreversible exchanges of matter and energy between the system and surroundings generate cyclical time in organisms. Shevek called this simultaneity because the endpoint of a cycle is the starting pointbeginning and end are the same. The overall effect of these cycles is maintenance, allowing living systems to persist long enough to be able to change. For biology, this is metabolic time, belonging to the ecological hierarchy. It is the cost of maintenance, of persisting; it is the cost of living. But cycles do not evolve; they only exist or disappear. For evolution to occur, biological systems must make linear time, which Shevek called sequency. Sequential time is made by irreversible thermodynamic production. This is what produces our sense of a distinction between past and future. For biological systems, this is the cost of moving forward, and it is cheap.

Blum (1968) evoked time as an arrow, creating a link between the passage of time and progress, time being driven forward into the future by power applied from "outside." But irreversibility requires only temporal asymmetry among the moving parts of an ensemble of components. Temporal asymmetry may occur as a result of some elements moving more slowly than others, interacting more often with fewer elements of the overall ensemble. This produces non-random correlations among the parts of the ensemble. Such historical correlations slow parts of the system, intensifying temporal asymmetries and thus irreversibility (in cosmological models, gravity performs this function). The distinction between ratchet irreversibility and temporal irreversibility is illusoryratchet irreversibility is simply the establishment of temporal correlations strong enough to limit the options the system can explore. Accumulated historical correlations produce irreversible system behavior regardless of the quantity or quality of novelty.

Or, as Maynard Smith and Szathmary (1995) stated, the conjunction of two or more events, each of which is improbable, is sufficient to make an evolutionary transition irreversible. History plays a central role here by maintaining improbable events long enough to increase the chances that two or more of them will become causally intertwined. A good example is self-reflexive nucleotide copying (DNA replication). The more faithful the replication, the higher the degree of historical correlation, the more stable the product, the slower the rate of evolutionary change. This produces historical inertia as a physical property of the nature of the organism. As the system grows and develops, it accumulates historical correlations that slow down matter and energy throughput, thereby decreasing the rate of entropy production and allowing for longer persistence (survival).

Because metabolism is inherited, it also plays a role in transitions from becoming to being, from existing to persisting, from persisting to evolving. This is the reason theories of the origin of life based on "metabolism only" produce persistent (proto) life but fail to produce evolvable life. Though expensive, simultaneity is not evolution; it is how we pay for evolution. Sequency is evolution. The temporal duality "buys" or "makes" enough time for "life" to be "evolvable life." It is far cheaper to evolve than to 
remain static - in fact, so much cheaper it is overwhelmingly probable that evolution will occur. McShea and Brandon (2011) called this a law of biology. But, as Ludwig von Boltzmann pointed out in 1905 , it is simply the biological manifestation of the Second Law of Thermodynamics in its statistical mechanical (probabilistic) form (Brooks 2011c).

\section{Space}

The distinction between the nature of the organism and the nature of the conditions creates a metaphorical spatial duality. The way in which organisms interact with themselves, embodied in the Genealogical Hierarchy, creates information space. The way in which organisms interact with their surroundings, embodied in the Ecological Hierarchy, creates fitness space.

\section{Information Space}

Informational space encompasses all possible material inheritances, including but not limited to genetic ones. It is in the nature of the organism to explore possible inheritances through reproduction and to expand information space through innovation. The realm of possible inheritances is Potential Information. The realm of actual inheritances is Realized Information. Evolutionary transitions are produced by innovations that increase the efficiency of storing and transmitting information. This allows both potential and realized information to grow simultaneously—realized evolutionary innovations always produce new potential innovations.

\section{Fitness Space}

Organisms impose themselves on their surroundings, changing them from "environment" to "fitness space." Each organism creates a kind of "fitness valley" in its surroundings analogous to gravity wells created by bodies in space/time. The space in which organisms have non-zero fitness is Fundamental Fitness Space. The portion of fundamental fitness space accessed is Realized Fitness Space. Genealogical conservatism (also called evolutionary lagload) provides a distinction between fundamental and realized fitness space proportional to selection strength. Realized fitness space may grow, but lagload will keep it a subset of fundamental fitness space. Evolutionary innovations may increase both fundamental and realized fitness space over time. When an organism dies, its fitness valley disappears. If the organism is replaced by another with the same requirements, the fitness valley may appear to persist, but it has no independent existence. The apparent persistence results from historical conservatism of the nature of the organism - to the extent that niches are real, they are products of the nature of the organism, not the nature of the conditions. Organisms thus (re)constitute niches; they do not construct them, nor are the surroundings inherently organized into niches.

\section{Complexity}

The evolutionary interaction of information space and fitness space produces prodigious amounts of evolutionary potential as both "spaces" expand over time. Organisms simultaneously inhabit both types of space, so doesn't Darwin's Necessary Mismatch dictate that the interaction of information and fitness space produces a restricted "evolutionary space"? The answer to this is as complex as the evolutionary process and depends on whether changes in the interactions are initiated by innovations in information space or the surroundings.

\section{The New Tangled Bank: Innovations in Information Space}

Evolutionary lagload means the genealogical system is incapable of being distributed at maximum density in all places at all times, so there will always be unoccupied or less-than-maximally occupied fitness space. The smaller the proportion of fitness space occupied, the "sloppier" it is (Agosta and Klemens 2008). As well, the "sloppiness" should increase over evolutionary time as the total information/fitness space grows, increasing the difference between what is possible and what is realized at any given time. Sloppy fitness space allows room for creativity and innovation because a lot of non-zero fitness space is always potentially available; however, sloppy fitness space does not rule out the possibility of local tightly optimized adaptations. Furthermore, sloppy fitness space allows the genealogical hierarchy to operate with a high degree of autonomy from the ecological hierarchy without sacrificing adaptability. The ability to move from surroundings that are deteriorating with respect to your fitness is more important in determining survival than how well adapted you are to any particular piece of the fitness space. And that ability (also called adaptability or resilience) is a function of how many historical alternatives you maintain in your collective genome (only successful adaptations get carried forward, so every bit of your history that you retain is a history of past success).

Evolutionary lagload also implies that there will be parts of fitness space where reproductive overrun creates conflicts from which there is no escape. These situations will be the foci of the most intense selection. Maynard Smith and Szathmary (1995) proposed that all major evolutionary transitions are associated with the origin of novel means of increasing the efficiency of storing and transmitting information; in other words, of major innovations in the genealogical system. Large-scale and novel innovations in the genealogical system should produce large-scale and novel interactions between information space and fitness space. This, in turn, should 
result in novel forms of selection. Brooks and Wiley (1988) used the term "cohesion" more or less synonymously with "transition" and proposed that all evolutionary innovations were characterized by the coupled evolution of novel forms of cohesion and selection. Eldredge's (1995) view emphasizes a hierarchy of selection processes emerging from the (co) evolution of the genealogical and ecological hierarchies.

\section{The Sloshing Bucket: Evolution Meets Climate Change}

Finally, evolutionary lagload implies the possibility of environmental changes of such a great magnitude or rapid rate that species as genealogical systems cannot cope and become extinct. Eldredge (2003) used the metaphor of a sloshing bucket for discussions of the macroevolutionary dynamics of environment-driven extinction and subsequent evolutionary renewal. In cases of great environmental perturbation (the bucket is sloshed so much that some of the contents are lost), portions of information space are eliminated, not just restricted in fitness space. As well, the nature of the conditions may also change. Both phenomena create new opportunities for the species that escape extinction.

Environmentally caused mass extinctions catalyze new speciation events. Extinctions in one area open up geographical territory for colonization by survivors, which initiates various forms of allopatric speciation. This alone, however, only increases the number of species - it does not necessarily increase evolutionary diversity. Fitness space gets sloppier as a result of the reduction of occupied information space, meaning that the range of non-zero fitness options for the survivors changes and grows. And that means increased opportunities for co-option of preexisting adaptations (McLennan 2008). If a now-extinct species had been keeping you out of part of your potential fitness space and that species goes extinct, this is called competitive release. If the environment changes in such a way that your fitness space is no longer marginal, it might appear that you have evolved a new adaptation. In reality, you will simply be showing off more of what you've had hidden in your stored evolutionary legacy. Having said all that, it is also true that such bucket-sloshing events help establish the conditions under which novel adaptations may arise, even if they do not actually initiate them. But most of the diversification is due to the rapid deployment of previously realized and potential information in previously inaccessible parts of fitness space, known by several names, such as taxon pulses, turnover pulses, and taxon cycles. This is the source of the decidedly punctuated aspect of the recovery following mass extinctions. As well, the more rapid the deployment of old and new information in fitness space, the more rapid the accumulation of historical correlations, slowing the process of diversification. This pattern has been interpreted by neo-Darwinians as an indication that the surroundings are constructed in such a way that only a certain number of species can be accommodated and as that magic number of species is approached, the rate of diversification slows. But the nature of species with respect to how their member organisms interact with their surroundings is an emergent property of the genealogical system, not the surroundings.

\section{Conclusions}

A friend recently cautioned that if I were too successful with my metaphors, no one would think I had said anything original. As I approach a stage of mature scholarship, I am not certain that I do have anything original to offer, except my enthusiasm for learning and the line of thought that has produced. I hope this contributes in some way to discussions that eventually produce the Extended Synthesis, and what will inevitably lie beyond that.

Finally, in keeping with the digital age, I close with four sound bites:

The Extended Synthesis in One Sentence: Increasing the autonomy of information flow enhances self-stability, which creates various forms of selection, which enhance mutual stability between the system and its surroundings.

The Extended Synthesis and The Fundamental Nature of Life: We're just recycled history machines. -Jimmy Buffett

The Extended Synthesis in One Metaphor: The more evolution occurs, the more evolution is possible.

The Extended Synthesis in One Cliche: Evolution is a journey, not a destination.

Acknowledgments Many have helped me throughout the past 30 years. For this contribution, I wish to give special thanks to Eors Szathmary, Niles Eldredge, Menachem Fisch, Christer Wiklund, Sven Jakobsson, Soren Nylin, Niklas Janz, Birgitta Tullberg, Sal Agosta, John Collier, and Deborah McLennan. Special thanks also to the staff and fellows of the Collegium Budapest, where I was honored to be a Senior Visiting Fellow in academic year 2010-2011.

\section{References}

Agosta SJ, Klemens JA. Ecological fitting by phenotypically flexible genotypes: implications for species associations, community assembly and evolution. Ecol Lett. 2008;11:1123-34.

Blum HF. Time's arrow and evolution. 3rd ed. Princeton: Princeton University Press; 1968.

Brooks DR. The Mastodon in the room: how Darwinian is neoDarwinism? Stud Hist Philos Biol Biomed Sci. 2011a;42:82-8.

Brooks DR. The extended synthesis: something old, something new. Evol Edu Outreach. 2011b;4:3-7.

Brooks DR. The extended synthesis: the law of the conditions of existence. Evol Edu Outreach. 2011c;4:254-61.

Brooks DR, Hoberg EP. Darwin's necessary misfit and the sloshing bucket: the evolutionary biology of emerging infectious diseases. Evol Edu Outreach. 2007;1:2-9.

Brooks DR, McLennan DA. The nature of diversity: an evolutionary voyage of discovery. Chicago: Univ. Chicago Press; 2002. 
Brooks DR, Wiley EO. Evolution as entropy: toward a unified theory of biology. 2nd ed. Chicago: University of Chicago Press; 1988.

Calcott, B, K Sterelny, editors. The major transitions in evolution revisited. Boston, MA: MIT Press; 2011.

Darwin C. The origin of species. 6th ed. London: John Murray; 1872.

Eldredge N. Reinventing Darwin: the great debate at the high table of evolutionary theory. New York: Wiley; 1995.

Eldredge N. The sloshing bucket: how the physical realm controls evolution. In: Crutchfield J, Schuster P, editors. Evolutionary dynamics. Exploring the interplay of selection, accident, neutrality, and function. SFI Studies in the Sciences of Complexity Series. New York: Oxford University Press; 2003. p. 3-32.
Fisch M, Benbaji Y. The view from within: normativity and the limits of self-criticism. South Bend: Univ. Notre Dame Press; 2011.

LeGuin U. The dispossessed. New York: Harper Collins; 1974.

Maynard Smith J, Szathmàry E. The major transitions in evolution. Oxford: W.H. Freeman; 1995.

Maynard Smith J, Szathmàry E. The origins of life. Oxford: Oxford University Press; 1999.

McLennan DA. The concept of co-option: why evolution often looks miraculous. Evol Educ Outreach. 2008;1:246-58.

McShea DW, Brandon RN. Biology's first law: the tendency for diversity and complexity to increase in evolutionary systems. Chicago: University of Chicago Press; 2011. 